\title{
ERRATUM, VOLUME 69
}

Albert Wilansky and Karl Zeller, $A$ biorthogonal system which is not a Toeplitz basis, pp. 725-726.

Include the statement "and $\left\{f_{n}\right\}$ is total" in the introduction and in the statement of Theorem 1. (Without this requirement an easy example can be given.) 